\title{
The Clinical Characteristics and Risk Factors of Severe COVID-19
}

\author{
Jianhua $\mathrm{Hu}^{\mathrm{a}}$ Yanggan Wang ${ }^{\mathrm{a}, \mathrm{b}}$ \\ aDepartment of Internal Medicine, Zhongnan Hospital of Wuhan University, Wuhan, China; \\ ${ }^{b}$ Medical Research Institute of Wuhan University, Wuhan, China
}

\section{Keywords}

Coronavirus disease $2019 \cdot$ Severe acute respiratory syndrome-CoV-2 · 2019-nCoV · Coronavirus · Pandemic

\begin{abstract}
Objective: We aim to investigate the clinical characteristics and risk factors for the severe cases of coronavirus disease 2019 (COVID-19) in comparison with the non-severe patients. Methods: We searched PubMed, EMBASE, Web of Science, and CNKI to collect all relevant studies published before July 26,2020 , and a total of 30 papers were included in this meta-analysis. Results: In the severe COVID-19 patients, $60 \%(95 \% \mathrm{Cl}=56-64 \%)$ were male, $25 \%(95 \% \mathrm{Cl}=21-29 \%)$ were over 65 years old, $34 \%(95 \% \mathrm{Cl}=24-44 \%)$ were obese, and $55 \%(95 \% \mathrm{Cl}=41-70 \%)$ had comorbidities. The most prevalent comorbidities were hypertension $(34 \%, 95 \% \mathrm{Cl}=$ $25-44 \%)$, diabetes $(20 \%, 95 \% \mathrm{Cl}=15-25 \%)$, and cardiovascular disease (CVD; $12 \%, 95 \% \mathrm{Cl}=9-16 \%)$. The most common blood test abnormalities were elevated C-reactive protein (CRP; 87\%, 82-92\%), decreased lymphocyte count (68\%, $58-77 \%)$, and increased lactate dehydrogenase $(69 \%, 95 \% \mathrm{Cl}$ $=57-81 \%)$. In addition, abnormal laboratory findings revealing organ dysfunctions were frequently observed in the severe cases, including decrease in albumin $43 \%, 95 \% \mathrm{Cl}=$ $24-63 \%)$ and increase in aspartate aminotransferase (47\%,
\end{abstract}

karger@karger.com

(c) 2021 S. Karger AG, Basel

www.karger.com/ger

Karger!
$95 \% \mathrm{Cl}=38-56 \%)$, alanine aminotransferase $(28 \%, 95 \% \mathrm{Cl}=$ 16-39\%), troponin l/troponin T (Tnl/TnT; 29\%, 95\% Cl = 13$45 \%)$, and serum $\mathrm{Cr}(\mathrm{SCr} ; 10 \%, 95 \% \mathrm{Cl}=5-15 \%)$. Conclusion: The male, elderly and obese patients and those with any comorbidities, especially with hypertension, diabetes, and CVD, were more likely to develop into severe cases. But the association between hypertension, diabetes, CVD, and severity of COVID-19 was declined by the increase of age. A significant elevation in cardiac Tnl/TnT, the hepatic enzymes, and $\mathrm{SCr}$ and the reduction in lymphocytes with elevated CRPs are important markers for the severity. Specific attention should be given to the elderly male and obese patients and those with indications of severe immune injury in combination with bacterial infection and indication of multi-organ dysfunction or damages.

(c) 2021 S. Karger AG, Basel

\section{Introduction}

In early December 2019, the coronavirus disease 2019 (COVID-19) broke out in Wuhan, China, and shortly reported over the world [1-4]. COVID-19 was caused by a previously unknown betacoronavirus, named 2019$\mathrm{nCoV}$ or severe acute respiratory syndrome (SARS)CoV-2 [5]. In this pandemic, the urban medical facilities 
in many countries have been overwhelmed with the treatment of severe cases [6]. Understanding the clinical characteristics and the risk factors of severe cases is critically important to improve the efficacy and outcome for the COVID-19 treatment. More and more studies have been published during the COVID-19 crisis. Here, we collected all relevant publications up to July 26, 2020, to reveal the clinical characteristics and the risk factors of severe COVID-19 to help identifying patients who are likely to develop to severe cases.

\section{Methods}

\section{Protocol and Search Strategy}

Our study followed PRISMA statement. We searched all relevant papers from PubMed, EMBASE, Web of Science, and CNKI with the following keywords: "2019-nCoV," "COVID-19," or "SARS-CoV-2" and "clinical characteristics," "clinical finding," "clinical feature," "clinical study," or "clinical case." The search period was updated to July 26, 2020. Then 2 researchers did a further artificial selection to screen eligible papers independently.

\section{The Inclusive and Exclusive Criteria}

In this article, we focused on the clinical characteristics of severe patients with COVID-19 and the differences between the severe and non-severe COVID-19 patients. The studies on observation of clinical characteristics in severe and non-severe patients with COVID-19 were included, and studies without severe patients' data or the comparison between the severe and non-severe patients or lack of clear diagnostic criteria were excluded.

The severe patients in our article included the patients hospitalized in intensive care unit and the severe/critical patients defined by the seventh guideline for the diagnosis and treatment of COVID-19 issued by the Chinese National Health Commission \& State Administration of Traditional Chinese Medicine. Because of the difficulty of obtaining original data, the classification was achieved based on the authors' statement presented in papers.

The severe illness of COVID-19 was defined if satisfying at least one of the following criteria: (a) breathing rate $\geq 30 / \mathrm{min}$; (b) pulse oximeter oxygen saturation $\leq 93 \%$ at rest; or (c) ratio of partial pressure of arterial oxygen to fraction of inspired oxygen $\leq 300 \mathrm{~mm}$ $\mathrm{Hg}(1 \mathrm{~mm} \mathrm{Hg}=0.133 \mathrm{kPa})$. Critical illness was defined if satisfying at least one of the following criteria: (a) respiratory failure with required mechanical ventilation; (b) shock; or (c) failure of other organs and received medical care in the intensive care unit. The reference intervals of laboratory findings varied in different studies, which was presented in online supplementary Table S1 (see www.karger.com/doi/10.1159/000513400 for all online suppl. material).

\section{Data Extraction}

The papers we searched and screened were imported to EndNote (version 8) to remove all duplicates. We then screened the titles and abstracts of retrieved papers in the list. All useful data were extracted in a form after reading the full text. Due to the reality in clinical practice and ethical restrictions, all included studies were cross-sectional studies.

\section{Statistical Analysis}

Based on the selected articles and available data, we did a singlearm meta-analysis to summarize the most common clinical characteristics in the severe patients with COVID-19 and another meta-analysis to compare the differences of clinical manifestations and laboratory findings between the severe and non-severe COVID-19 patients. We used R (version 3.6.1) to calculate pooled estimated prevalence with $95 \%$ confidence intervals of basic characteristics, comorbidities, and laboratory findings, and Stata (version 12.0) was used to calculated OR and 95\% CI to estimate every indicator of each paper. A random-effect model was used to calculate the combined ORs and its 95\% CI. Cochran's Q test and $I^{2}$ statistics were used to evaluate the heterogeneity. Random-effects meta-regression was conducted to assess the effect of age on the association of comorbidities with severity of COVID-19. Sensitivity analyses were used to evaluate risk of bias and stability of the results. Egger's test was used to assess publication bias in which $p<0.05$ indicated a significant publication bias.

\section{Results}

\section{Literature Summary}

We retrieved a total of 637 papers. 292 repetitive papers were removed and 131 papers were excluded after screening the titles and abstracts. 182 articles were eliminated due to lack of a comparison between non-severe and severe COVID-19 patients, short of original data or diagnosis criteria. At the end, 30 papers were included in our meta-analysis [1], [7-35]. The screening process was shown in Figure 1. The detailed information about basic characteristics, underlying diseases, and laboratory findings was summarized in Tables 1 and 2 and online supplementary Tables S1 and S2.

Because of the reality in clinical practice and medical ethics, all included studies are case series studies. A total of 6,685 individuals were involved in our study with the sample size ranging from 41 to 1,099 .

\section{Meta-Analysis Results}

The Clinical Characteristics of Severe COVID-19

A total of 1,457 severe COVID-19 patients were included in this meta-analysis. In the severe COVID-19 patients, $60 \%(95 \% \mathrm{CI}=56-64 \%)$ were male, $25 \%(95 \%$ $\mathrm{CI}=21-29 \%)$ were over 65 years old, $34 \%(95 \% \mathrm{CI}=$ $24-44 \%)$ were obese, and 55\% (95\% CI $=41-70 \%)$ had comorbidities. The most prevalent comorbidities were hypertension $(34 \%, 95 \% \mathrm{CI}=25-44 \%)$, diabetes $(20 \%$, 95\% CI $=15-25 \%$ ), and cardiovascular disease (CVD; $12 \%, 95 \% \mathrm{CI}=9-16 \%)$. For laboratory findings, the prevalence of elevated C-reactive protein (CRP) was $87 \%$ (82$92 \%)$, decreased lymphocyte count was $68 \%$ (58-77\%), and increased lactate dehydrogenase (LDH) was $69 \%$ 
Fig. 1. Flow diagram of the retrieval pro-

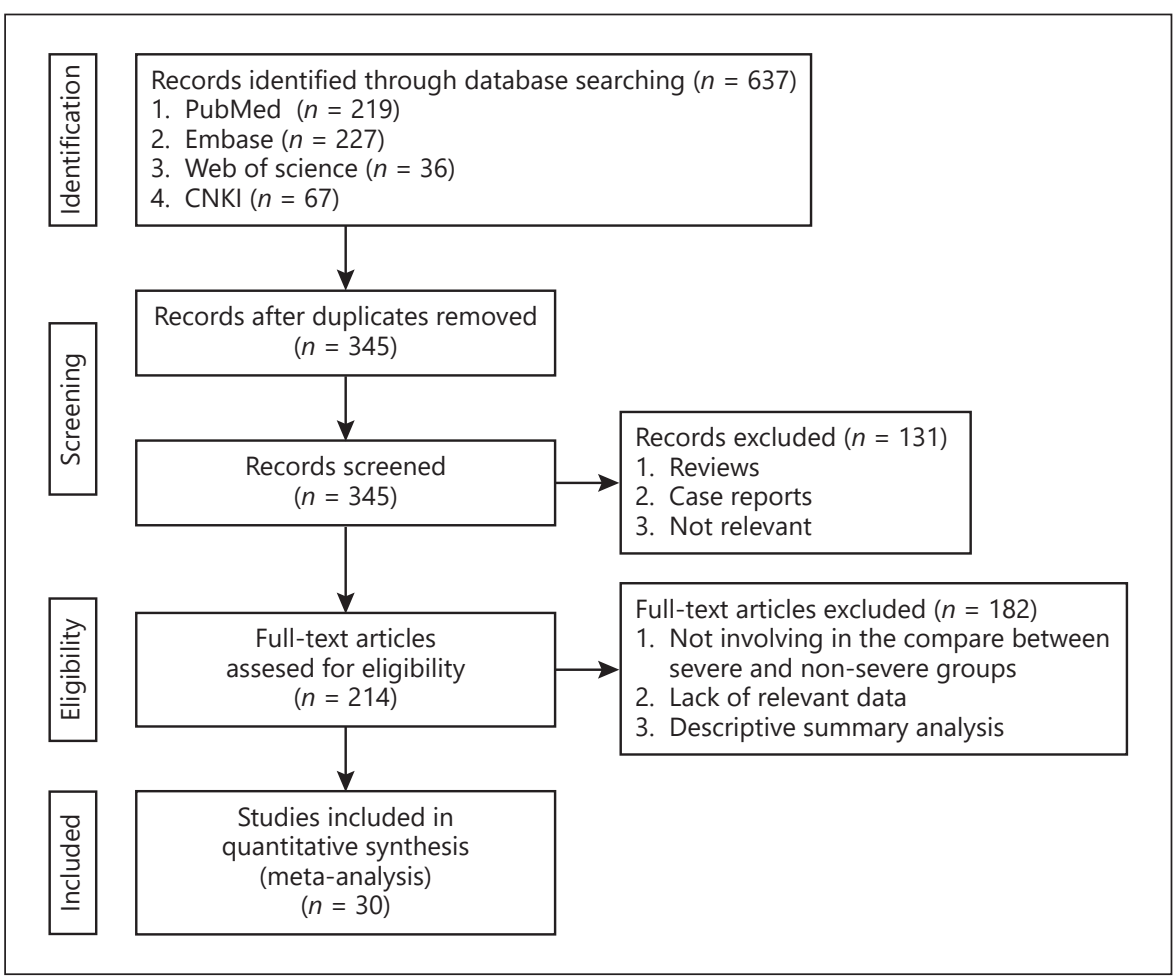

(95\% CI $=57-81 \%)$. In addition, significant decrease in albumin $(43 \%, 95 \% \mathrm{CI}=24-63 \%)$ and increase in aspartate aminotransferase (AST; 47\%, 95\% CI $=38-56 \%$ ), alanine aminotransferase (ALT; 28\%, 95\% CI $=16-39 \%$ ), troponin I/troponin $\mathrm{T}(\mathrm{TnI} / \mathrm{TnT}$; $29 \%, 95 \% \mathrm{CI}=13-$ $45 \%)$, and serum $\mathrm{Cr}$ (SCr; 10\%, 95\% CI $=5-15 \%)$ were observed (Table 3 ).

The Differences of Clinical Characteristics between Severe and Non-severe Groups

As shown in Table 4, male $(\mathrm{OR}=1.383,95 \% \mathrm{CI}=$ $1.183-1.616)$, age over 65 years old $(\mathrm{OR}=2.250,95 \%$ $\mathrm{CI}=1.677-3.017)$, and obesity $(\mathrm{OR}=2.519,95 \% \mathrm{CI}=$ 1.498-4.235) were associated with increased severity of COVID-19. The prevalence of comorbidity, such as hypertension, CVD, and diabetes in the severe patients was significantly higher $(\mathrm{OR}=2.661,95 \% \mathrm{CI}=1.700-4.163$; $\mathrm{OR}=2.041,95 \% \mathrm{CI}=1.591-2.619 ; \mathrm{OR}=2.264,95 \%$ $\mathrm{CI}=1.705-3.007 ; \mathrm{OR}=2.156,95 \% \mathrm{CI}=1.651-2.815$ ) than non-severe cases. In the severe patients, increase in white blood cell count $(\mathrm{OR}=2.784,95 \% \mathrm{CI}=1.878$ $4.125)$ and decrease in lymphocyte $(\mathrm{OR}=2.054,95 \%$ $\mathrm{CI}=1.641-2.571)$ and platelet count $(\mathrm{OR}=1.852,95 \%$ $\mathrm{CI}=1.602-2.142)$ were common. Furthermore, the incidence rate of decreased albumin $(\mathrm{OR}=2.257,95 \% \mathrm{CI}=$
1.562-3.262) and increased levels of CRP (OR $=1.495$, $95 \% \mathrm{CI}=1.353-1.652)$, procalcitonin $(\mathrm{OR}=2.403,95 \%$ $\mathrm{CI}=1.727-3.343)$, AST $(\mathrm{OR}=2.356,95 \% \mathrm{CI}=1.917-$ 2.896), ALT (OR $=1.857,95 \% \mathrm{CI}=1.389-2.483), \mathrm{LDH}$ $(\mathrm{OR}=1.744,95 \% \mathrm{CI}=1.384-2.199), \mathrm{TnI} / \mathrm{TnT}(\mathrm{OR}=$ $4.707,95 \% \mathrm{CI}=2.234-9.917)$, and $\mathrm{SCr}(\mathrm{OR}=2.245,95 \%$ $\mathrm{CI}=1.474-3.421)$ was significantly higher in the severe patients.

The Result of Meta-regression

The meta-regression analysis with age as a covariate indicated that age declined the association between hypertension $(p=0.027)$, diabetes $(p=0.001), \operatorname{CVD}(p=$ $0.003)$, and severity of COVID-19.

\section{Heterogeneity Test, Sensitivity Analysis and} Publication Bias

We observed significant heterogeneity $\left(I^{2}\right)$ varying from 52.1 to $96.2 \%$ in meta-analysis of the clinical characteristics of severe COVID-19 (Table 3). Significant publication bias $(p<0.05)$ was observed in the prevalence of CVD, increased white blood cells, increased CRP, and increased serum $\mathrm{Cr}$ (as shown in Table 3).

As for meta-analysis of the differences between severe and non-severe patients, heterogeneity varied from 0.0 to 


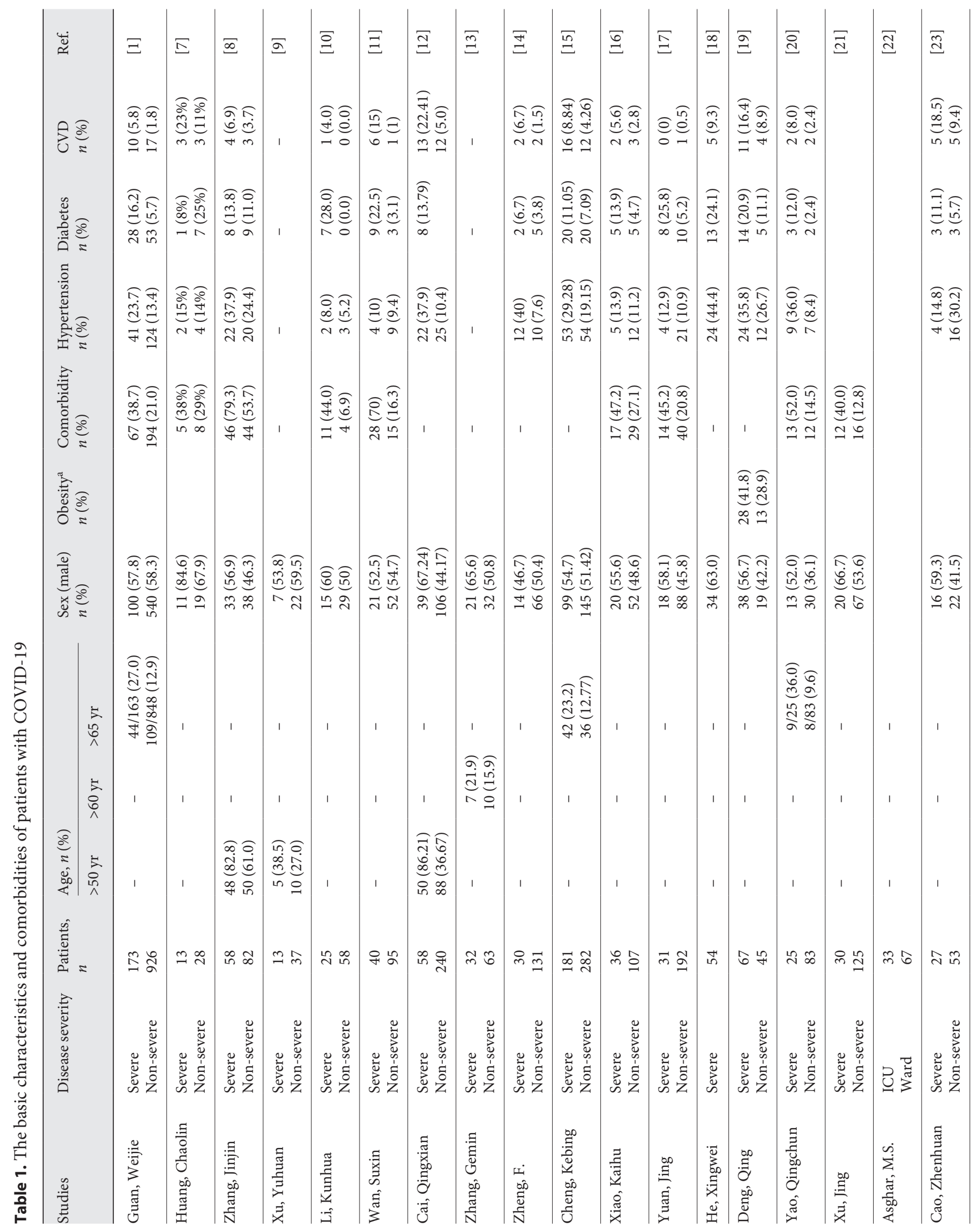




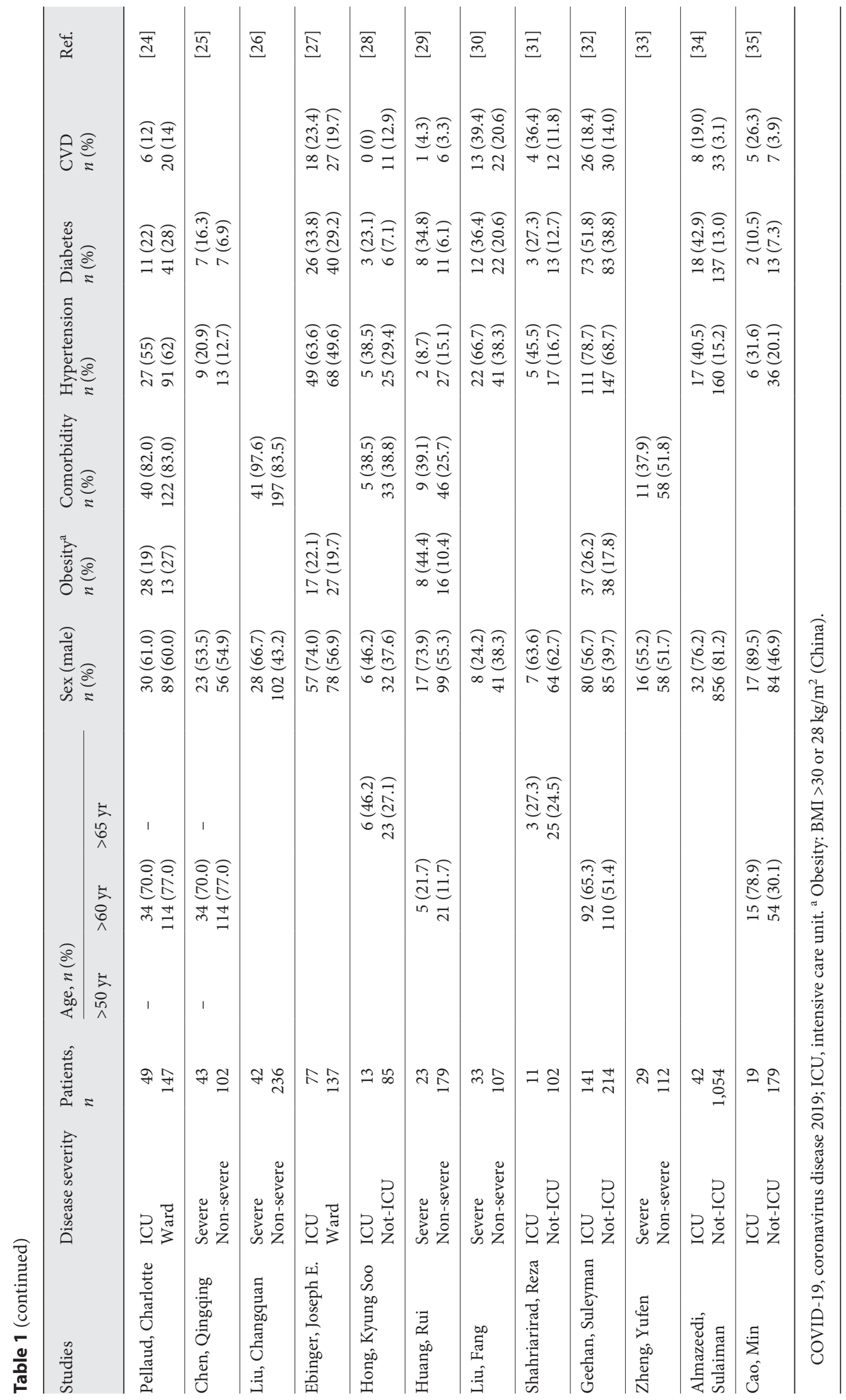




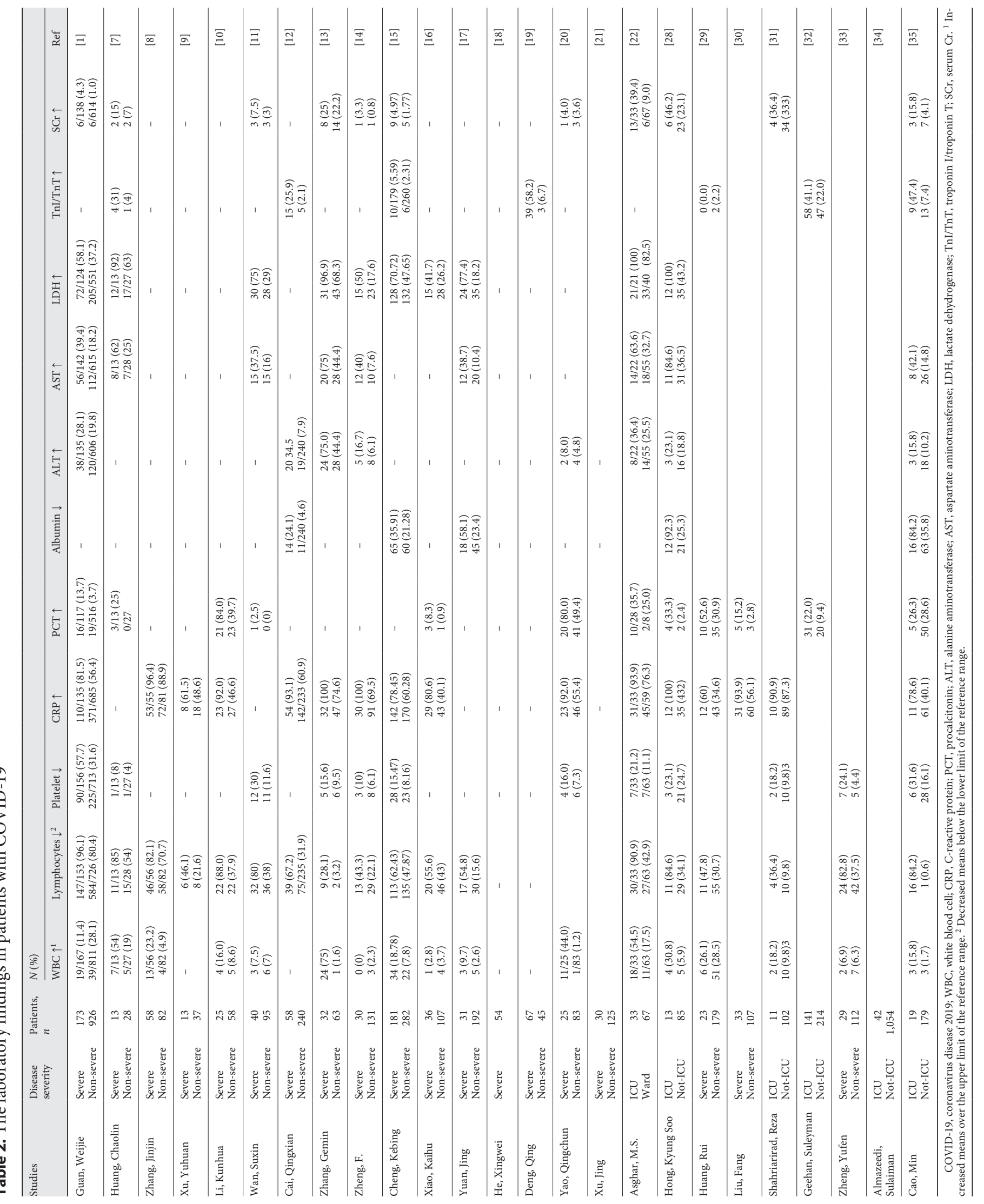


Table 3. The clinical characteristics of severe patients with COVID-19

\begin{tabular}{|c|c|c|c|c|c|c|}
\hline Clinical characteristic & $\begin{array}{l}\text { Studies, } \\
n\end{array}$ & Subgroup & Prevalence & $95 \% \mathrm{CI}$ & $\begin{array}{l}\text { Quantifying } \\
\text { heterogeneity, \% }\end{array}$ & Egger test \\
\hline Male & 29 & $\begin{array}{l}\text { Total } \\
\text { Severe } \\
\text { ICU }\end{array}$ & $\begin{array}{l}0.60 \\
0.58 \\
0.65\end{array}$ & $\begin{array}{l}{[0.56 ; 0.64]} \\
{[0.55 ; 0.61]} \\
{[0.60 ; 0.70]}\end{array}$ & $\begin{array}{l}52.1 \\
45.9 \\
59.1\end{array}$ & 0.9551 \\
\hline Age & $\begin{array}{l}3 \\
5 \\
4\end{array}$ & $\begin{array}{l}>50 \mathrm{yr} \\
>60 \mathrm{yr} \\
>65 \mathrm{yr}\end{array}$ & $\begin{array}{l}0.74 \\
0.52 \\
0.25\end{array}$ & $\begin{array}{l}{[0.57 ; 0.92]} \\
{[0.30 ; 0.73]} \\
{[0.21 ; 0.29]}\end{array}$ & $\begin{array}{r}82.3 \\
92.5 \\
0.0\end{array}$ & 0.7839 \\
\hline Obesity & 6 & $\begin{array}{l}\text { Total } \\
\text { Severe } \\
\text { ICU }\end{array}$ & $\begin{array}{l}0.34 \\
0.35 \\
0.34\end{array}$ & $\begin{array}{l}{[0.24 ; 0.44]} \\
{[0.26 ; 0.44]} \\
{[0.17 ; 0.52]}\end{array}$ & $\begin{array}{l}77.7 \\
19.5 \\
89.2\end{array}$ & 0.1885 \\
\hline Comorbidity & 15 & $\begin{array}{l}\text { Total } \\
\text { Severe } \\
\text { ICU }\end{array}$ & $\begin{array}{l}0.55 \\
0.54 \\
0.60\end{array}$ & $\begin{array}{l}{[0.41 ; 0.70]} \\
{[0.37 ; 0.72]} \\
{[0.32 ; 0.87]}\end{array}$ & $\begin{array}{l}95.1 \\
95.9 \\
83.4\end{array}$ & 0.4874 \\
\hline Hypertension & 24 & $\begin{array}{l}\text { Total } \\
\text { Severe } \\
\text { ICU }\end{array}$ & $\begin{array}{l}0.34 \\
0.28 \\
0.52\end{array}$ & $\begin{array}{l}{[0.25 ; 0.44]} \\
{[0.21 ; 0.34]} \\
{[0.38 ; 0.67]}\end{array}$ & $\begin{array}{l}93.2 \\
82.6 \\
86.3\end{array}$ & 0.0963 \\
\hline Diabetes & 26 & $\begin{array}{l}\text { Total } \\
\text { Severe } \\
\text { ICU }\end{array}$ & $\begin{array}{l}0.20 \\
0.15 \\
0.31\end{array}$ & $\begin{array}{l}{[0.15 ; 0.25]} \\
{[0.12 ; 0.19]} \\
{[0.19 ; 0.43]}\end{array}$ & $\begin{array}{l}81.8 \\
52.2 \\
82.8\end{array}$ & 0.0523 \\
\hline CVD & 24 & $\begin{array}{l}\text { Total } \\
\text { Severe } \\
\text { ICU }\end{array}$ & $\begin{array}{l}0.12 \\
0.11 \\
0.17\end{array}$ & $\begin{array}{l}{[0.09 ; 0.16]} \\
{[0.07 ; 0.14]} \\
{[0.10 ; 0.24]}\end{array}$ & $\begin{array}{l}74.8 \\
72.3 \\
65.1\end{array}$ & 0.0017 \\
\hline WBC increased & 17 & $\begin{array}{l}\text { Total } \\
\text { Severe } \\
\text { ICU }\end{array}$ & $\begin{array}{l}0.22 \\
0.20 \\
0.30\end{array}$ & $\begin{array}{l}{[0.15 ; 0.30]} \\
{[0.12 ; 0.28]} \\
{[0.10 ; 0.50]}\end{array}$ & $\begin{array}{l}90.5 \\
91.4 \\
74.8\end{array}$ & 0.0134 \\
\hline Lymphocyte decreased & 20 & $\begin{array}{l}\text { Total } \\
\text { Severe } \\
\text { ICU }\end{array}$ & $\begin{array}{l}0.68 \\
0.65 \\
0.78\end{array}$ & $\begin{array}{l}{[0.58 ; 0.77]} \\
{[0.54 ; 0.77]} \\
{[0.60 ; 0.95]}\end{array}$ & $\begin{array}{l}92.2 \\
93.5 \\
76.3\end{array}$ & 0.269 \\
\hline Platelet decreased & 14 & $\begin{array}{l}\text { Total } \\
\text { Severe } \\
\text { ICU }\end{array}$ & $\begin{array}{l}0.20 \\
0.19 \\
0.23\end{array}$ & $\begin{array}{l}{[0.11 ; 0.30]} \\
{[0.08 ; 0.31]} \\
{[0.14 ; 0.33]}\end{array}$ & $\begin{array}{r}89.0 \\
92.3 \\
0.0\end{array}$ & 0.0789 \\
\hline CRP increased & 18 & $\begin{array}{l}\text { Total } \\
\text { Severe } \\
\text { ICU }\end{array}$ & $\begin{array}{l}0.87 \\
0.87 \\
0.92\end{array}$ & $\begin{array}{l}{[0.82 ; 0.92]} \\
{[0.81 ; 0.92]} \\
{[0.86 ; 0.98]}\end{array}$ & $\begin{array}{r}82.0 \\
86.0 \\
0.0\end{array}$ & 0.0055 \\
\hline PCT increased & 12 & $\begin{array}{l}\text { Total } \\
\text { Severe } \\
\text { ICU }\end{array}$ & $\begin{array}{l}0.32 \\
0.34 \\
0.24\end{array}$ & $\begin{array}{l}{[0.19 ; 0.44]} \\
{[0.15 ; 0.53]} \\
{[0.18 ; 0.30]}\end{array}$ & $\begin{array}{r}93.1 \\
95.5 \\
0.0\end{array}$ & 0.1314 \\
\hline LDH increased & 11 & $\begin{array}{l}\text { Total } \\
\text { Severe } \\
\text { ICU }\end{array}$ & $\begin{array}{l}0.69 \\
0.67 \\
0.78\end{array}$ & $\begin{array}{l}{[0.57 ; 0.81]} \\
{[0.53 ; 0.81]} \\
{[0.50 ; 1.00]}\end{array}$ & $\begin{array}{l}91.8 \\
93.0 \\
84.8\end{array}$ & 0.1586 \\
\hline Albumin decreased & 5 & $\begin{array}{l}\text { Total } \\
\text { Severe } \\
\text { ICU }\end{array}$ & $\begin{array}{l}0.43 \\
0.50 \\
0.38\end{array}$ & $\begin{array}{l}{[0.24 ; 0.63]} \\
{[0.0 ; 1]} \\
{[0.23 ; 0.53]}\end{array}$ & $\begin{array}{l}91.5 \\
96.4 \\
81.1\end{array}$ & 1 \\
\hline AST increased & 10 & $\begin{array}{l}\text { Total } \\
\text { Severe } \\
\text { ICU }\end{array}$ & $\begin{array}{l}0.47 \\
0.43 \\
0.56\end{array}$ & $\begin{array}{l}{[0.38 ; 0.56]} \\
{[0.35 ; 0.50]} \\
{[0.29 ; 0.84]}\end{array}$ & $\begin{array}{l}66.9 \\
40.0 \\
83.6\end{array}$ & 0.0892 \\
\hline ALT increased & 9 & $\begin{array}{l}\text { Total } \\
\text { Severe } \\
\text { ICU }\end{array}$ & $\begin{array}{l}0.28 \\
0.31 \\
0.21\end{array}$ & $\begin{array}{l}{[0.16 ; 0.39]} \\
{[0.15 ; 0.45]} \\
{[0.11 ; 0.31]}\end{array}$ & $\begin{array}{r}86.1 \\
91.0 \\
0.0\end{array}$ & 0.8348 \\
\hline TnI/TnT increased & 7 & $\begin{array}{l}\text { Total } \\
\text { Severe } \\
\text { ICU }\end{array}$ & $\begin{array}{l}0.29 \\
0.23 \\
0.42\end{array}$ & $\begin{array}{l}{[0.13 ; 0.45]} \\
{[0.06 ; 0.40]} \\
{[0.34 ; 0.49]}\end{array}$ & $\begin{array}{r}96.2 \\
95.6 \\
0.0\end{array}$ & 0.2931 \\
\hline SCr increased & 10 & $\begin{array}{l}\text { Total } \\
\text { Severe } \\
\text { ICU }\end{array}$ & $\begin{array}{l}0.10 \\
0.06 \\
0.29\end{array}$ & $\begin{array}{l}{[0.05 ; 0.15]} \\
{[0.03 ; 0.08]} \\
{[0.13 ; 0.46]}\end{array}$ & $\begin{array}{l}70.6 \\
31.1 \\
52.8\end{array}$ & 0.0095 \\
\hline
\end{tabular}

COVID-19, coronavirus disease 2019; CVD, cardiovascular disease; WBC, white blood cell; CRP, C-reactive protein; PCT, procalcitonin; ALT, alanine aminotransferase; AST, aspartate aminotransferase; LDH, lactate dehydrogenase; TnI/TnT, troponin $\mathrm{I} /$ troponin T; SCr, serum Cr.

The Clinical Characteristics and Risk Factors of Severe COVID-19 
Table 4. The association of different patients' characteristics and clinical manifestations with increased severity of COVID-19

\begin{tabular}{|c|c|c|c|c|c|c|}
\hline Clinical characteristic & Subgroup & Pooled OR & $95 \%$ CI & $I^{2}, \%$ & $p$ value & Egger test \\
\hline Male & $\begin{array}{l}\text { Total } \\
\text { Severe } \\
\text { ICU }\end{array}$ & $\begin{array}{l}1.383 \\
1.306 \\
1.405\end{array}$ & $\begin{array}{l}1.183 ; 1.616 \\
1.144 ; 1.618 \\
0.964 ; 2.047\end{array}$ & $\begin{array}{l}22.3 \\
18.7 \\
39.0\end{array}$ & $\begin{array}{l}0.000 \\
0.001 \\
0.077\end{array}$ & 0.385 \\
\hline Age & $\begin{array}{l}>50 \mathrm{yr} \\
>60 \mathrm{yr} \\
>65 \mathrm{yr}\end{array}$ & $\begin{array}{l}4.153 \\
1.841 \\
2.250 \\
\end{array}$ & $\begin{array}{l}1.426 ; 12.090 \\
0.905 ; 3.746 \\
1.677 ; 3.017\end{array}$ & $\begin{array}{r}73.8 \\
72.8 \\
0.0\end{array}$ & $\begin{array}{l}0.009 \\
0.092 \\
0.000\end{array}$ & $\begin{array}{l}0.650 \\
0.442 \\
0.343\end{array}$ \\
\hline Obesity & $\begin{array}{l}\text { Total } \\
\text { Severe } \\
\text { ICU }\end{array}$ & $\begin{array}{l}2.519 \\
2.380 \\
1.267\end{array}$ & $\begin{array}{l}1.498 ; 4.235 \\
0.995 ; 5.695 \\
0.706 ; 2.276\end{array}$ & $\begin{array}{l}81.7 \\
71.6 \\
89.8\end{array}$ & $\begin{array}{l}0.000 \\
0.051 \\
0.000\end{array}$ & 0.198 \\
\hline Comorbidity & $\begin{array}{l}\text { Total } \\
\text { Severe } \\
\text { ICU }\end{array}$ & $\begin{array}{l}2.661 \\
3.258 \\
1.267 \\
\end{array}$ & $\begin{array}{l}1.700 ; 4.163 \\
1.955 ; 5.428 \\
0.706 ; 2.276\end{array}$ & $\begin{array}{r}71.5 \\
72.8 \\
8.2 \\
\end{array}$ & $\begin{array}{l}0.000 \\
0.000 \\
0.427 \\
\end{array}$ & 0.289 \\
\hline Hypertension & $\begin{array}{l}\text { Total } \\
\text { Severe } \\
\text { ICU }\end{array}$ & $\begin{array}{l}2.041 \\
2.170 \\
1.835 \\
\end{array}$ & $\begin{array}{l}1.591 ; 2.619 \\
1.586 ; 2.970 \\
1.188 ; 2.833\end{array}$ & $\begin{array}{l}50.1 \\
48.1 \\
56.8\end{array}$ & $\begin{array}{l}0.000 \\
0.000 \\
0.006\end{array}$ & 0.545 \\
\hline CVD & $\begin{array}{l}\text { Total } \\
\text { Severe } \\
\text { ICU }\end{array}$ & $\begin{array}{l}2.264 \\
2.353 \\
2.056\end{array}$ & $\begin{array}{l}1.705 ; 3.007 \\
1.815 ; 3.051 \\
1.061 ; 3.983\end{array}$ & $\begin{array}{r}44.4 \\
79.1 \\
0.0\end{array}$ & $\begin{array}{l}0.000 \\
0.000 \\
0.033\end{array}$ & 0.148 \\
\hline Diabetes & $\begin{array}{l}\text { Total } \\
\text { Severe } \\
\text { ICU }\end{array}$ & $\begin{array}{l}2.156 \\
2.569 \\
1.608\end{array}$ & $\begin{array}{l}1.651 ; 2.815 \\
1.906 ; 3.463 \\
1.043 ; 1.043\end{array}$ & $\begin{array}{l}63.7 \\
36.1 \\
76.8\end{array}$ & $\begin{array}{l}0.000 \\
0.000 \\
0.032\end{array}$ & 0.030 \\
\hline WBC increased & $\begin{array}{l}\text { Total } \\
\text { Severe } \\
\text { ICU }\end{array}$ & $\begin{array}{l}2.784 \\
2.530 \\
3.573\end{array}$ & $\begin{array}{l}1.878 ; 4.125 \\
1.546 ; 4.142 \\
2.201 ; 5.800\end{array}$ & $\begin{array}{r}55.2 \\
61.4 \\
0.1\end{array}$ & $\begin{array}{l}0.000 \\
0.000 \\
0.000\end{array}$ & 0.069 \\
\hline Lymphocyte decreased & $\begin{array}{l}\text { Total } \\
\text { Severe } \\
\text { ICU }\end{array}$ & $\begin{array}{l}2.054 \\
1.851 \\
4.262\end{array}$ & $\begin{array}{l}1.641 ; 2.571 \\
1.496 ; 2.289 \\
1.817 ; 9.997\end{array}$ & $\begin{array}{l}91.7 \\
89.9 \\
88.2\end{array}$ & $\begin{array}{l}0.000 \\
0.000 \\
0.001\end{array}$ & 0.094 \\
\hline Platelet decreased & $\begin{array}{l}\text { Total } \\
\text { Severe } \\
\text { ICU }\end{array}$ & $\begin{array}{l}1.852 \\
1.871 \\
1.680\end{array}$ & $\begin{array}{l}1.602 ; 2.142 \\
1.607 ; 2.178 \\
1.037 ; 2.720\end{array}$ & $\begin{array}{l}0.0 \\
0.0 \\
0.0\end{array}$ & $\begin{array}{l}0.000 \\
0.000 \\
0.035\end{array}$ & 0.635 \\
\hline CRP increased & $\begin{array}{l}\text { Total } \\
\text { Severe } \\
\text { ICU }\end{array}$ & $\begin{array}{l}1.495 \\
1.493 \\
1.523\end{array}$ & $\begin{array}{l}1.353 ; 1.652 \\
1.345 ; 1.658 \\
1.087 ; 2.132\end{array}$ & $\begin{array}{l}79.3 \\
77.8 \\
87.4\end{array}$ & $\begin{array}{l}0.000 \\
0.000 \\
0.014\end{array}$ & 0.009 \\
\hline PCT increased & $\begin{array}{l}\text { Total } \\
\text { Severe } \\
\text { ICU }\end{array}$ & $\begin{array}{l}2.403 \\
2.613 \\
2.151\end{array}$ & $\begin{array}{l}1.727 ; 3.343 \\
1.753 ; 3.894 \\
0.930 ; 4.973\end{array}$ & $\begin{array}{l}54.7 \\
57.2 \\
68.3\end{array}$ & $\begin{array}{l}0.000 \\
0.000 \\
0.073\end{array}$ & 0.002 \\
\hline Albumin decreased & $\begin{array}{l}\text { Total } \\
\text { Severe } \\
\text { ICU }\end{array}$ & $\begin{array}{l}2.257 \\
2.555 \\
1.352\end{array}$ & $\begin{array}{l}1.562 ; 3.262 \\
1.501 ; 4.350 \\
0.260 ; 7.029\end{array}$ & $\begin{array}{l}69.9 \\
77.2 \\
83.5\end{array}$ & $\begin{array}{l}0.000 \\
0.001 \\
0.720\end{array}$ & 0.585 \\
\hline ALT increased & $\begin{array}{l}\text { Total } \\
\text { Severe } \\
\text { ICU }\end{array}$ & $\begin{array}{l}1.857 \\
2.057 \\
1.407 \\
\end{array}$ & $\begin{array}{l}1.389 ; 2.483 \\
1.396 ; 3.032 \\
0.830 ; 2.384\end{array}$ & $\begin{array}{r}41.1 \\
60.6 \\
0.0 \\
\end{array}$ & $\begin{array}{l}0.000 \\
0.000 \\
0.205\end{array}$ & 0.645 \\
\hline AST increased & $\begin{array}{l}\text { Total } \\
\text { Severe } \\
\text { ICU }\end{array}$ & $\begin{array}{l}2.356 \\
2.468 \\
2.292 \\
\end{array}$ & $\begin{array}{l}1.917 ; 2.896 \\
1.810 ; 3.363 \\
1.756 ; 2.990 \\
\end{array}$ & $\begin{array}{r}39.1 \\
56.6 \\
0.0 \\
\end{array}$ & $\begin{array}{l}0.000 \\
0.000 \\
0.000 \\
\end{array}$ & 0.087 \\
\hline $\mathrm{LDH}$ increased & $\begin{array}{l}\text { Total } \\
\text { Severe } \\
\text { ICU }\end{array}$ & $\begin{array}{l}1.744 \\
1.860 \\
1.315 \\
\end{array}$ & $\begin{array}{l}1.384 ; 2.199 \\
1.487 ; 2.326 \\
0.443 ; 3.901 \\
\end{array}$ & $\begin{array}{l}86.2 \\
81.5 \\
96.3 \\
\end{array}$ & $\begin{array}{l}0.000 \\
0.000 \\
0.622 \\
\end{array}$ & 0.243 \\
\hline SCr increased & $\begin{array}{l}\text { Total } \\
\text { Severe } \\
\text { ICU }\end{array}$ & $\begin{array}{l}2.245 \\
2.003 \\
2.568 \\
\end{array}$ & $\begin{array}{l}1.474 ; 3.421 \\
1.247 ; 3.217 \\
0.971 ; 6.792 \\
\end{array}$ & $\begin{array}{r}21.2 \\
0.0 \\
66.8 \\
\end{array}$ & $\begin{array}{l}0.000 \\
0.000 \\
0.057 \\
\end{array}$ & 0.534 \\
\hline $\operatorname{Tn} \mathrm{I} / \operatorname{TnT}$ increased & $\begin{array}{l}\text { Total } \\
\text { Severe } \\
\text { ICU }\end{array}$ & $\begin{array}{l}4.707 \\
6.034 \\
3.357\end{array}$ & $\begin{array}{l}2.234 ; 9.917 \\
2.758 ; 13.201 \\
0.978 ; 11.520\end{array}$ & $\begin{array}{l}77.2 \\
40.5 \\
90.2\end{array}$ & $\begin{array}{l}0.000 \\
0.000 \\
0.054\end{array}$ & 0.154 \\
\hline
\end{tabular}

COVID-19, coronavirus disease 2019; CVD, cardiovascular disease; WBC, white blood cell; CRP, C-reactive protein; PCT, procalcitonin; ALT, alanine aminotransferase; AST, aspartate aminotransferase; LDH, lactate dehydrogenase; $\operatorname{TnI} / \mathrm{TnT}$, troponin I/troponin T; SCr, serum Cr. 
91.7\% (Table 4). Significant publication bias $(p<0.05)$ was observed in the prevalence of diabetes, increased CRP and procalcitonin (as shown in Table 4).

Sensitivity analysis was conducted by excluding any study, and the significant results remained unchanged. Detailed results of Egger's test were shown in Tables 3 and 4.

In summary of the meta-analysis results, the male, elderly, obese people, and patients with any comorbidities, especially with hypertension, diabetes, and CVD, were more likely to develop into severe cases. A significant percentage of severe patients had multi-organ dysfunctions or damages, including the damage of immune system.

\section{Discussion/Conclusion}

The original host of this virus is still not identified, although bats are the most possible suspect. A structural analysis study found that the SARS-CoV-2 external receptor-binding domain was similar to that of SARS-CoV. The angiotensin-converting enzyme 2 (ACE2) receptor in human cells is considered to be the binding site of SARS-CoV-2, which is also the cellular receptor for SARS coronavirus [36-38]. Further studies demonstrated that SARS-CoV-2 employed ACE2 for cell entry through the binding of the viral spike $(S)$ proteins to cellular receptor ACE2 [39].

ACE2 is distributed in multiple organs, including the lungs, heart, kidneys, liver, and intestine [40-43]. Several studies found that in addition to the respiratory system, the damages in other systems might also be related to SARS-CoV-2 attack. Zheng et al. [44] reported that SARSCoV-2 was responsible for acute cardiac injury and maybe mediated by ACE2. In addition, Xiao et al. [45] reported that SARS-CoV-2 could infect the cytoplasm of gastric, duodenal, and rectum glandular epithelial cell. Also, the liver impairment might be directly caused by the viral infection or due to drug hepatotoxicity [46]. Furthermore, SARS-CoV-2 infection also promotes secretion of inflammatory cytokines and systemic inflammatory response, and in some cases leading to inflammatory storm. A recent autopsy revealed the deep airway inflammation, alveoli damage, exudative inflammation, and the hydropericardium and myocardial changes due to SARS-CoV-2 infection or pre-existing CVD were also observed [47]. These findings indicate that SARS-CoV-2 not only induces lung injury but also may cause systematic damages in COVID-19 patients.

The patients with CVD deserve particular attention. Li et al. [48] concluded that there was a significant associa-

The Clinical Characteristics and Risk

Factors of Severe COVID-19 tion between COVID-19 mortality and cardiac injury. A study found that ACE2 activity is increased in patients with type 1 diabetes with vascular complications [49]. It is known that the ACE2 expression and activity are connected with Angiotensin-converting enzyme inhibitor (ACEI). ACEI is the drug for treating hypertension and heart failure by regulating blood pressure and prevention of ventricular remodeling via suppressing the elevated activity of renin-angiotensin-aldosterone system. During ACEI application, ACE2 activity is not inhibited. Instead, the upregulation of ACE2 expression and increased activity were observed $[50,51]$. However, latest studies found that ACEI or ARB use was not associated with more severe COVID-19 disease [52], but the use of ARBs increased the risk of SARS-CoV-2 infection in younger patients [53].

Our study found that patients with hypertension, diabetes, and CVD are more sensitive to SARS-CoV-2 infection and transition to severity. However, the association between hypertension, diabetes, and CVD and severity of COVID-19 was decreased by age. It is likely that age itself is closely related with the propensity of comorbidities which contributes somewhat to the severity transition.

Also, male patients are more sensitive to the infection of bacteria, virus, parasite, and fungi [54]. This may be linked to their living habits. For instance, there are much more smokers in men than in women. In a recent metaanalysis study [55], Vardavas and Nikitara [55] reported that the smokers were 1.4 times more likely to have severe symptoms of COVID-19 and 2.4 times more likely to be admitted to an intensive care unit, need mechanical ventilation, or die compared to nonsmokers. This may be linked to an increased ACE2 gene expression in the smokers. From another point of review, in the smokers, unlikely to wear a mask and frequent hand-to-mouth contact may also increase the risk of SARS-CoV-2 infection. Given these, the male seems more easily to be attacked by SARS-CoV-2 and more likely to transit into severe cases.

In addition, obesity is a main risk factor of comorbidities such as hypertension, diabetes mellitus, and CVD [56]. Meanwhile, ACE2 abundantly expressed in adipose tissue. These may cause obese people vulnerable to SARSCoV-2 as well [57].

COVID-19 patients were in some cases complicated with myocarditis. However, the diagnosis of myocarditis was largely based on troponin elevation. Actually, the myocardial injury could be likely caused by the viral infection-triggered inflammatory response, rather than the direct viral attack, and there was no SARS-CoV-2 observed in the heart tissue [58-61]. 
In terms of nervous injury, most publications included in our study only observed nonspecific neurological symptoms, such as headache, dizziness, and agitation. More specific manifestations like delirium were not generally reported. Mao et al. [62] and Julie Helms et al. [63] found delirium and/or neurological symptoms appeared more frequent in severe COVID-19 patients and were associated with worse prognosis. However, the direct evidence of COVID-19 invasion to nervous system is still limited. The RT-PCR test of SARS-CoV-2 was negative in cerebrospinal fluid and there was not remarkable pathological observation in the brain $[61,63]$.

Hypoalbuminemia in critically ill patients was statistically significant and associated with longer hospitalization and higher mortality [64]. Decreased albumin levels in severe COVID-19 patients could be the results of liver dysfunction or malnutrition due to gastrointestinal symptoms [65]. But hypoalbuminemia is a predictor of transition to the severity independent of age and comorbidity [66].

Comparing COVID-19 with SARS or Middle East Respiratory Syndrome, age, male gender, and comorbidities such as diabetes and hypertension were the mortality risk factors in common $[67,68]$. Besides, laboratory findings including decreased lymphocytes, platelet count, and albumin level and increased AST, LDH, and CRP levels in patients diagnosed with COVID-19 were not remarkably different from those of patients diagnosed with SARS or Middle East respiratory syndrome [69].

We have to notice that the abnormal laboratory variants were obtained at the time of hospitalization but not always collected prior to the patient transition to the severe cases. In some cases, the results may be collected in patients who had already become a severe case at the time of hospital admission. Under this circumstance, we cannot rule out the possibility that these abnormalities were secondary to the disease development (present in online suppl. Tables S1, S2).

In summary, our study revealed that the elderly, male, obese people, and patients with any comorbidities, espe- cially those with hypertension, CVD, or diabetes are more likely to develop to severe cases. A progressive elevation in cardiac TnI/TnT, the hepatic enzymes, and serum $\mathrm{Cr}$ and the advanced lymphocytopenia and leukocytosis are important alerting markers of mild to severe case transition.

A total of 30 papers were included in this meta-analysis. Although the literatures included in this study have good quality, some limitations remain. First, due to the inconsistency of the observation time of each study, heterogeneity and bias are inevitable. Second, all studies included in this meta-analysis are retrospective studies, and all the study objects were inpatients diagnosed with SARS-CoV-2 infection. Therefore, many patients who did not go to hospital were not included. A more solid conclusion can be achieved when more well-designed large-scale clinical trial studies become available.

\section{Statement of Ethics}

This study is exempt from Ethical Committee Approval since all human data were collected from the published sources.

\section{Conflict of Interest Statement}

The authors have no conflicts of interest to declare.

\section{Funding Sources}

This work was supported by grants awarded to Yanggan Wang from the National Natural Science Foundation of China (NSFC, Grant Nos. 81873507 and 81420108004 ).

\section{Author Contributions}

The research idea and study design were guided by Yanggan Wang. The data extraction and analysis were done by Jianhua Hu. The manuscript was written by Jianhua $\mathrm{Hu}$ and modified by Yanggan Wang.

\section{References}

1 Guan WJ, Ni ZY, Hu Y, Liang WH, Ou CQ, $\mathrm{He}$ JX, et al. Clinical characteristics of coronavirus disease 2019 in China. N Engl J Med. 2020;382(18):1708-20.

2 Holshue ML, DeBolt C, Lindquist S, Lofy KH, Wiesman J, Bruce H, et al. First case of 2019 novel coronavirus in the United States. N Engl J Med. 2020;382(10):929-36.
3 Phan T. Novel coronavirus: from discovery to clinical diagnostics. Infect Genet Evol. 2020; 79:104211.

4 Rothe C, Schunk M, Sothmann P, Bretzel G, Froeschl G, Wallrauch C, et al. Transmission of 2019-nCoV infection from an asymptomatic contact in Germany. N Engl J Med. 2020; 382(10):970-1.
5 Yang X, Yu Y, Xu J, Shu H, Xia J, Liu H, et al. Clinical course and outcomes of critically ill patients with SARS-CoV-2 pneumonia in Wuhan, China: a single-centered, retrospective, observational study. Lancet Respir Med. 2020;8(5):475-81. 
6 Lipsitch M, Swerdlow DL, Finelli L. Defining the epidemiology of covid-19: studies needed. N Engl J Med. 2020;382(13):1194-6.

7 Huang C, Wang Y, Li X, Ren L, Zhao J, Hu Y, et al. Clinical features of patients infected with 2019 novel coronavirus in Wuhan, China. Lancet. 2020;395(10223):497-506.

8 Zhang J-J, Dong X, Cao Y-Y, Yuan Y-D, Yang Y-B, Yan Y-Q, et al. Clinical characteristics of 140 patients infected with SARS-CoV-2 in Wuhan, China. Allergy. 2020 Jul;75(7):173041.

$9 \mathrm{Xu}$ YH, Dong JH, An WM, Lv XY, Yin XP, Zhang JZ, et al. Clinical and computed tomographic imaging features of novel coronavirus pneumonia caused by SARS-CoV-2. J Infect. 2020;80(4):394-400.

10 Li K, Wu J, Wu F, Guo D, Chen L, Fang Z, et al. The clinical and chest CT features associated with severe and critical COVID-19 pneumonia. Invest Radiol. 2020;55(6):327-31.

11 Wan S, Xiang Y, Fang W, Zheng Y, Li B, Hu $\mathrm{Y}$, et al. Clinical features and treatment of COVID-19 patients in northeast Chongqing. J Med Virol. 2020 Jul;92(7):797-806.

12 Cai Q, Huang D, Ou P, Yu H, Zhu Z, Xia Z, et al. COVID-19 in a designated infectious diseases hospital outside Hubei Province, China. Allergy. 2020 Jul;75(7):1742-52.

13 Zhang G, Zhang J, Wang B, Zhu X, Wang Q, Qiu S. Analysis of clinical characteristics and laboratory findings of 95 cases of 2019 novel coronavirus pneumonia in Wuhan, China: a retrospective analysis. Respir Res. 2020;21(1): 74.

14 Zheng F, Tang W, Li H, Huang YX, Xie YL, Zhou ZG. Clinical characteristics of 161 cases of corona virus disease 2019 (COVID-19) in Changsha. Eur Rev Med Pharmacol Sci. 2020; 24(6):3404-10

15 Cheng K, Wei M, Shen H, Wu C, Chen D, Xiong W, et al. Clinical characteristics of 463 patients with common and severe type coronavirus disease 2019. Shanghai Med J. 2020: $1-15$.

16 Xiao K, Shui L, Pang X, Huaming M, Wang J, Lang $\mathrm{C}$, et al. The clinical features of the 143 patients with COVID-19 in North-East of Chongqing. J Third Mil Med Univ. 2020:1-5.

17 Yuan J, Ya-yu S, Yu-jie Z, Chen T-y, Cao Q, Yuan G-d, et al. A retrospective analysis of the clinical characteristics of $223 \mathrm{NCP}$ patients in Chongqing. J Southwest Univ. 2020;42(3): $17-24$.

18 He XW, Lai JS, Cheng J, Wang MW, Liu YJ, Xiao ZC, et al. Impact of complicated myocardial injury on the clinical outcome of severe or critically ill COVID-19 patients. Zhonghua Xin Xue Guan Bing Za Zhi. 2020;48:E011.

19 Deng Q, Hu B, Zhang Y, Wang H, Zhou X, Hu $\mathrm{W}$, et al. Suspected myocardial injury in patients with COVID-19: evidence from frontline clinical observation in Wuhan, China. Int J Cardiol. 2020;130(5):390-9.

20 Yao Q, Wang P, Wang X, Qie G, Meng M, Tong X, et al. A retrospective study of risk factors for severe acute respiratory syndrome coronavirus 2 infections in hospitalized adult patients. Pol Arch Intern Med. 2020;130(5): 390-9.

$21 \mathrm{Xu} \mathrm{J}$, Zhao F, Han M, Ma L, Zhang T. [Analysis of the clinical characteristics and early warning model construction of severe/critical coronavirus disease 2019 patients]. Zhonghua Wei Zhong Bing Ji Jiu Yi Xue. 2020;32(4): 401-6.

22 Asghar MS, Haider Kazmi SJ, Ahmed Khan N, Akram M, Ahmed Khan S, Rasheed U, et al. Clinical profiles, characteristics, and outcomes of the first 100 admitted COVID-19 patients in Pakistan: a single-center retrospective study in a Tertiary Care Hospital of Karachi. Cureus. 2020;12(6):e8712.

23 Cao Z, Li T, Liang L, Wang H, Wei F, Meng S, et al. Clinical characteristics of Coronavirus Disease 2019 patients in Beijing, China. PLoS One. 2020;15(6): $\mathrm{e} 0234764$.

24 Pellaud C, Grandmaison G, Pham Huu Thien HP, Baumberger M, Carrel G, Ksouri H, et al. Characteristics, comorbidities, 30-day outcome and in-hospital mortality of patients hospitalised with COVID-19 in a Swiss area: a retrospective cohort study. Swiss Med Wkly. 2020;150:w20314.

25 Chen Q, Zheng Z, Zhang C, Zhang X, Wu $\mathrm{H}$, Wang J, et al. Clinical characteristics of 145 patients with corona virus disease 2019 (COVID-19) in Taizhou, Zhejiang, China. Infection. 2020:1-9.

26 Liu C, Deng X, Pan Y, Ling Z, Zhang G, Wei $\mathrm{G}$, et al. [Clinical characteristics and CT imaging features of patients with different clinical types of coronavirus disease 2019]. Zhonghua Wei Zhong Bing Ji Jiu Yi Xue. 2020;32(5): 548-53.

27 Ebinger JE, Achamallah N, Ji H, Claggett BL, Sun N, Botting P, et al. Pre-existing traits associated with Covid-19 illness severity. PLoS One. 2020;15(7):e0236240.

28 Hong KS, Lee KH, Chung JH, Shin KC, Choi $\mathrm{EY}$, Jin HJ, et al. Clinical features and outcomes of 98 patients hospitalized with SARSCoV-2 infection in Daegu, South Korea: a brief descriptive study. Yonsei Med J. 2020; 61(5):431-7.

29 Huang R, Zhu L, Xue L, Liu L, Yan X, Wang J, et al. Clinical findings of patients with coronavirus disease 2019 in Jiangsu province, China: a retrospective, multi-center study. PLoS Negl Trop Dis. 2020;14(5):e0008280.

30 Liu F, Li L, Xu M, Wu J, Luo D, Zhu Y, et al. Prognostic value of interleukin-6, C-reactive protein, and procalcitonin in patients with COVID-19. J Clin Virol. 2020;127:104370.

31 Shahriarirad R, Khodamoradi Z, Erfani A, Hosseinpour H, Ranjbar K, Emami Y, et al. Epidemiological and clinical features of 2019 novel coronavirus diseases (COVID-19) in the South of Iran. BMC Infect Dis. 2020;20(1): 427.

32 Suleyman G, Fadel RA, Malette KM, Hammond C, Abdulla H, Entz A, et al. Clinical characteristics and morbidity associated with coronavirus disease 2019 in a series of patients in metropolitan detroit. JAMA Netw Open. 2020;3(6):e2012270.

33 Zheng Y, Zhang Y, Chi H, Chen S, Peng M, Luo L, et al. The hemocyte counts as a potential biomarker for predicting disease progression in COVID-19: a retrospective study. Clin Chem Lab Med. 2020;58(7):1106-15.

34 Almazeedi S, Al-Youha S, Jamal MH, AlHaddad M, Al-Muhaini A, Al-Ghimlas F, et al. Characteristics, risk factors and outcomes among the first consecutive 1096 patients diagnosed with COVID-19 in Kuwait. EClinicalMedicine. 2020;24:100448.

35 Cao M, Zhang D, Wang Y, Lu Y, Zhu X, Li Y, et al. Clinical features of patients infected with the 2019 Novel Coronavirus (COVID-19) in Shanghai, China. medRxiv. 2020 Mar 6:

36 Ge XY, Li JL, Yang XL, Chmura AA, Zhu G Epstein $\mathrm{JH}$, et al. Isolation and characterization of a bat SARS-like coronavirus that uses the ACE2 receptor. Nature. 2013;503(7477): 535-8.

37 Lu R, Zhao X, Li J, Niu P, Yang B, Wu H, et al Genomic characterisation and epidemiology of 2019 novel coronavirus: implications for virus origins and receptor binding. Lancet. 2020;395(10224):565-74.

38 Yan R, Zhang Y, Li Y, Xia L, Guo Y, Zhou Q. Structural basis for the recognition of SARSCoV-2 by full-length human ACE2. Science. 2020;367(6485):1444-8

39 Hoffmann M, Kleine-Weber H, Schroeder S, Krüger N, Herrler T, Erichsen S, et al. SARSCoV-2 cell entry depends on ACE2 and TMPRSS2 and is blocked by a clinically proven protease inhibitor. Cell. 2020;181(2):271e8.

40 Danilczyk U, Penninger JM. Angiotensinconverting enzyme II in the heart and the kidney. Circ Res. 2006;98(4):463-71.

41 Donoghue M, Hsieh F, Baronas E, Godbout K, Gosselin M, Stagliano N, et al. A novel angiotensin-converting enzyme-related carboxypeptidase (ACE2) converts angiotensin I to angiotensin 1-9. Circ Res. 2000;87(5):E19.

42 Paizis G, Tikellis C, Cooper ME, Schembri JM, Lew RA, Smith AI, et al. Chronic liver injury in rats and humans upregulates the novel enzyme angiotensin converting enzyme 2. Gut. 2005;54(12):1790-6.

43 Turner AJ, Hiscox JA, Hooper NM. ACE2: from vasopeptidase to SARS virus receptor. Trends Pharmacol Sci. 2004;25(6):291-4.

44 Zheng YY, Ma YT, Zhang JY, Xie X. COVID-19 and the cardiovascular system. Nat Rev Cardiol. 2020;17(5):259-60.

45 Xiao F, Tang M, Zheng X, Liu Y, Li X, Shan H. Evidence for gastrointestinal infection of SARS-CoV-2. Gastroenterology. 2020;158(6): 1831-33.e3.

46 Zhang C, Shi L, Wang F-S. Liver injury in COVID-19: management and challenges. Lancet Gastroenterol Hepatol. 2020;5(5):428-30.

47 Qian L, Rong-shuai W, Guo-qiang Q. Gross examination report of a COVID-19 death autopsy. J Forensic Med. 2020;36(1):21-3.
The Clinical Characteristics and Risk Factors of Severe COVID-19 
48 Li J-W, Han T-W, Woodward M, Anderson CS, Zhou H, Chen Y-D, et al. The impact of 2019 novel coronavirus on heart injury: a systematic review and meta-analysis. Prog Cardiovasc Dis. 2020 Jul-Aug;63(4):518-24.

49 Soro-Paavonen A, Gordin D, Forsblom C, Rosengard-Barlund M, Waden J, Thorn L, et al. Circulating ACE2 activity is increased in patients with type 1 diabetes and vascular complications. J Hypertens. 2012;30(2):37583.

50 Arendse LB, Danser AHJ, Poglitsch M, Touyz RM, Burnett JC, Llorens-Cortes C, et al. Novel therapeutic approaches targeting the reninangiotensin system and associated peptides in hypertension and heart failure. Pharmacol Rev. 2019;71(4):539-70.

51 Ferrario CM, Jessup J, Chappell MC, Averill DB, Brosnihan KB, Tallant EA, et al. Effect of angiotensin-converting enzyme inhibition and angiotensin ii receptor blockers on cardiac angiotensin-converting enzyme 2. Circulation. 2005;111(20):2605-10.

52 Mackey K, King VJ, Gurley S, Kiefer M, Liederbauer E, Vela K, et al. Risks and impact of angiotensin-converting enzyme inhibitors or angiotensin-receptor blockers on SARSCoV-2 infection in adults: a living systematic review. Ann Intern Med. 2020;173(3):195203.

53 Chan C-K, Huang Y-S, Liao H-W, Tsai IJ, Sun $\mathrm{C}-\mathrm{Y}, \mathrm{Pan} \mathrm{H}-\mathrm{C}$, et al. Renin-angiotensin-aldosterone system inhibitors and risks of SARSCoV-2 infection: a systematic review and meta-analysis. Hypertension. 2020 Nov;76(5): 1563-71.

54 Jaillon S, Berthenet K, Garlanda C. Sexual dimorphism in innate immunity. Clinic Rev Allerg Immunol. 2019;56(3):308-21.
55 Vardavas C, Nikitara K. COVID-19 and smoking: a systematic review of the evidence. Tob Induc Dis. 2020 Mar 20;18:20.

56 Stefan N, Birkenfeld AL, Schulze MB, Ludwig DS. Obesity and impaired metabolic health in patients with COVID-19. Nat Rev Endocrinol. 2020;16(7):341-2.

57 Jia XY C, Lu S, Chen Y, Liu Q, Bai J, Lu Y. Two things about COVID-19 might need attention. Preprints. 2020;2020:2020020315.

58 Fox SE, Akmatbekov A, Harbert JL, Li G, Quincy Brown J, Vander Heide RS. Pulmonary and cardiac pathology in African American patients with COVID-19: an autopsy series from New Orleans. Lancet Respir Med. 2020;8(7):681-6.

59 Xu Z, Shi L, Wang Y, Zhang J, Huang L, Zhang C, et al. Pathological findings of COVID-19 associated with acute respiratory distress syndrome. Lancet Respir Med. 2020; 8(4):420-2.

60 Sala S, Peretto G, Gramegna M, Palmisano A, Villatore A, Vignale D, et al. Acute myocarditis presenting as a reverse Tako-Tsubo syndrome in a patient with SARS-CoV-2 respiratory infection. Eur Heart J. 2020;41(19): 1861-

61 Bradley BT, Maioli H, Johnston R, Chaudhry I, Fink SL, Xu H, et al. Histopathology and ultrastructural findings of fatal COVID-19 infections in Washington State: a case series. Lancet. 2020;396(10247):320-32.

62 Mao L, Jin $\mathrm{H}$, Wang M, Hu Y, Chen S, He Q, et al. Neurologic manifestations of hospitalized patients with Coronavirus Disease 2019 in Wuhan, China. JAMA Neurol. 2020;77(6): 683-90.
63 Helms J, Kremer S, Merdji H, Schenck M, Severac F, Clere-Jehl R, et al. Delirium and encephalopathy in severe COVID-19: a cohort analysis of ICU patients. Crit Care. 2020; 24(1):491.

64 de la Rica R, Borges M, Aranda M, Del Castillo A, Socias A, Payeras A, et al. Low albumin levels are associated with poorer outcomes in a case series of COVID-19 patients in Spain: a retrospective cohort study. Microorganisms. 2020;8(8):1106.

65 Zarifian A, Zamiri Bidary M, Arekhi S, Rafiee M, Gholamalizadeh H, Amiriani A, et al. Gastrointestinal and hepatic abnormalities in patients with confirmed COVID-19: a systematic review and meta-analysis. J Med Virol. 2020 Jul 18.

66 Huang J, Cheng A, Kumar R, Fang Y, Chen G, Zhu Y, et al. Hypoalbuminemia predicts the outcome of COVID-19 independent of age and co-morbidity. J Med Virol. 2020 Oct; 92(10):2152-8.

67 Pormohammad A, Ghorbani S, Khatami A, Farzi R, Baradaran B, Turner DL, et al. Comparison of confirmed COVID-19 with SARS and MERS cases: clinical characteristics, laboratory findings, radiographic signs and outcomes: a systematic review and meta-analysis. Rev Med Virol. 2020;30(4):e2112.

68 Lu L, Zhong W, Bian Z, Li Z, Zhang K, Liang $\mathrm{B}$, et al. A comparison of mortality-related risk factors of COVID-19, SARS, and MERS: a systematic review and meta-analysis. J Infect. 2020 Oct;81(4):e18-25.

69 Petrosillo N, Viceconte G, Ergonul O, Ippolito G, Petersen E. COVID-19, SARS and MERS: are they closely related? Clin Microbiol Infect. 2020;26(6):729-34. 